\title{
BERTMeSH: Deep Contextual Representation Learning for Large-scale High-performance MeSH Indexing with Full Text
}

\author{
Ronghui You ${ }^{1}$, Yuxuan Liu ${ }^{1}$, Hiroshi Mamitsuka ${ }^{2,3}$ and Shanfeng Zhu ${ }^{4,5,1 *}$ \\ ${ }^{1}$ School of Computer Science and Shanghai Key Lab of Intelligent Information Processing, Fudan University, Shanghai 200433, China. \\ ${ }^{2}$ Bioinformatics Center, Institute for Chemical Research, Kyoto University, Uji, Kyoto Prefecture, Japan. ${ }^{3}$ Department of Computer \\ Science, Aalto University, Espoo, Finland. ${ }^{4}$ Institute of Science and Technology for Brain-Inspired Intelligence and Shanghai Institute of \\ Artificial Intelligence Algorithms, Fudan University, Shanghai 200433, China. ${ }^{5}$ Ministry of Education, Key Laboratory of Computational \\ Neuroscience and Brain-Inspired Intelligence (Fudan University), China
}

*To whom correspondence should be addressed.

\begin{abstract}
Motivation: With the rapid increase of biomedical articles, large-scale automatic Medical Subject Headings $(\mathrm{MeSH})$ indexing has become increasingly important. FullMeSH, the only method for largescale MeSH indexing with full text, suffers from three major drawbacks: FullMeSH 1) uses Learning To Rank (LTR), which is time-consuming, 2) can capture some pre-defined sections only in full text, and 3) ignores the whole MEDLINE database.

Results: We propose a computationally lighter, full-text and deep learning based MeSH indexing method, BERTMeSH, which is flexible for section organization in full text. BERTMeSH has two technologies: 1) the state-of-the-art pre-trained deep contextual representation, BERT (Bidirectional Encoder Representations from Transformers), which makes BERTMeSH capture deep semantics of full text. 2) a transfer learning strategy for using both full text in PubMed Central (PMC) and title and abstract (only and no full text) in MEDLINE, to take advantages of both. In our experiments, BERTMeSH was pre-trained with 3 million MEDLINE citations and trained on approximately 1.5 million full text in PMC. BERTMeSH outperformed various cutting edge baselines. For example, for $20 \mathrm{~K}$ test articles of PMC, BERTMeSH achieved a Micro F-measure of $69.2 \%$, which was $6.3 \%$ higher than FullMeSH with the difference being statistically significant. Also prediction of $20 \mathrm{~K}$ test articles needed 5 minutes by BERTMeSH, while it took more than 10 hours by FullMeSH, proving the computational efficiency of BERTMeSH.

Contact: zhusf@fudan.edu.cn
\end{abstract}

\section{Introduction}

As a comprehensive controlled vocabulary, Medical Subject Headings (MeSH) has been developed and maintained by the National Library of Medicine (NLM) for indexing, cataloging and searching of biomedical information (Sayers et al., 2020). As of 2020, there are 29,640 MeSH main headings $(\mathrm{MHs})^{1}$. One of the most important usages of MeSH is to index the largest biomedical literature database, MEDLINE, which currently covers more than 5,200 journals and 26 million citations (Sayers et al.,

${ }^{1}$ https://www.nlm.nih.gov/databases/download/mesh.html
2020). Currently each MEDLINE citation is annotated with 13 MHs on average, which can be utilized in many applications in biomedical text mining and information retrieval (Lu et al., 2009; Stokes et al., 2009; Gu et al., 2013; Huang et al., 2011; Zhu et al., 2009). Accurate MeSH indexing is thus crucial for biomedical researchers, who are generating new hypotheses and seeking to make new discoveries.

In 2019, 956,390 citations have been added into MEDLINE, which is around 5\% increase over $2018(904,636)^{2}$. The vast majority of these citations are manually indexed with MHs by human curators in NLM with an average annotation cost of $\$ 9.4$ per citation (Mork et al., 2013). To

${ }^{2}$ https://www.nlm.nih.gov/bsd/medline_pubmed_production_stats.html 
deal with the rapid growth of MEDLINE, NLM has developed a software tool, Medical Text Indexer (MTI), to facilitate the MeSH indexing task in automated and semi-automated modes (Aronson et al., 2004; Mork et al., 2017). Currently around 5\% of MEDLINE citations are annotated automatically, where MTI provides MHs without human intervention ${ }^{3}$. On the other hand, around $18 \%$ of MEDLINE citations are annotated semiautomatically, where human curators review (and possibly revise) the MHs recommended by MTI. Note that MTIs use only the title and abstract of each citation to recommend MHs, while human curators in NLM check the full text to finish the MeSH indexing task. Meanwhile, the number of available full text in PubMed Central (PMC) reaches 5.9 million in Jan $2020{ }^{4}$. With the rapid growth of full text biomedical articles, it is an imperative task to develop an accurate and efficient automatic MeSH indexing method for large-scale full text.

From a machine learning perspective, automatic MeSH indexing can be deemed as a large-scale multi-label learning problem, where MHs are labels, citations are instances, and each citation is associated with multiple MHs (Liu et al., 2015). To advance the performance of automatic MeSH indexing, many advanced machine learning methods have been developed to address this challenging problem in the last few years, such as MetaLabeler (Tsoumakas et al., 2013), MeSHNow (Mao and Lu, 2017), MeSHLabeler (Liu et al., 2015), DeepMeSH (Peng et al., 2016), AttentionMeSH (Jin et al., 2018), MeSHProbeNet (Xun et al., 2019) and FullMeSH (Dai et al., 2020). Different from all other methods using title and abstract only, FullMeSH makes use of full text to extract different sections, and utilizes Learning To Rank (LTR)(Li, 2011) to integrate the evidence generated from each section to improve the performance of MeSH indexing. However, FullMeSH suffers from three major drawbacks: 1) the performance of FullMeSH drops significantly if pre-defined sections are missed in the full text. This is because FullMeSH relies on pattern matching to extract five standard sections: Title and Abstract, Introduction, Methods and Materials, Result and Experiment, Conclusion and Summary. Many biomedical articles however do not have all these five sections. Additionally, pre-defined patterns can hardly deal with all kinds of variations of section names. 2) FullMeSH relies on LTR to integrate many different types of evidence generated from each section, which is complicated, laborious and time consuming. 3) although FullMeSH uses the full text of PMC open access data to train the model, FullMeSH cannot take advantage of the whole MEDLINE database.

In this work, we propose a novel deep learning based method, BERTMeSH, to improve the performance of large-scale MeSH indexing with full text. The main contributions of BERTMeSH are as follows:

- To the best of our knowledge, BERTMeSH is the first end-to-end deep learning based automatic MeSH indexing method for full text of largescale biomedical documents $(>1 \mathrm{M})$ and all $29 \mathrm{k}$ MHs. In contrast to FullMeSH of LTR, BERTMeSH adopts a deep multi-label model with attention mechanism to capture the most relevant part of text for each label. In addition, we use Bidirectional Encoder Representations from Transformers (BERT) (Devlin et al., 2019) in BERTMeSH to encode the input text. In general natural language processing (NLP), we can find many successful applications of BERT, which can better capture text context and semantics in NLP. As BERTMeSH is an end-to-end deep model, it is more convenient for the developer to train, test, deploy and maintain the model.

- Without relying on pattern matching to distinguish different sections, BERTMeSH can robustly use the content of full text. Besides the title and abstract, the top four longest sections of each full text biomedical article are extracted as input in BERTMeSH. This avoids the missing

\footnotetext{
$\overline{3 \text { https://www.nlm.nih.gov/pubs/techbull/ja18/ja18_indexing_method.htm }}$

${ }^{4}$ https://www.ncbi.nlm.nih.gov/pmc
}

section problem, caused by pattern matching and heterogeneous nature of different articles.

- In addition to utilizing full text in PMC, BERTMeSH can take advantage of the whole MEDLINE to improve the performance of large-scale MeSH indexing. The MEDLINE database is utilized in two distinct ways: 1) the whole MEDLINE has been used as corpus to train BERT model (BioBERT(Lee et al., 2020)), which can better reflect the characteristics of biomedical articles, and is used to encode text in BERTMeSH. 2) the network parameters in BERTMeSH are pre-trained by millions of MEDLINE citations, which greatly boost the performance.

- We conducted a thorough experiment for validating BERTMeSH by using PMC Open Access Subset (>1.4M) with 20,000 test articles. BERTMeSH achieved Micro F-measure of $69.2 \%$, being $6.3 \%$ and $6.0 \%$ higher than those of the two start-of-the-art MeSH indexing methods, FullMeSH (65.1, trained on whole PMC Open Access Subset) and DeepMeSH (65.3, training on whole MEDLINE), respectively. Furthermore, BERTMeSH improves around $8.3 \%$ in Micro F-measure over FullMeSH for indexing full text articles with at least one missing section.

\section{Related Work}

\subsection{Large-scale MeSH indexing based on title and abstract}

To the best of our knowledge, all state-of-the-art large MeSH indexing methods, except FullMeSH, use title and abstract only. A classic method for large-scale MeSH indexing is NLM-developed MTI (Aronson et al., 2004; Mork et al., 2017) with two components: PubMed-Related citations (PRC) and MetaMap Indexing (MMI). PRC is a modified $k$-nearest neighbor $(\mathrm{KNN})$ algorithm, to obtain the $\mathrm{MHs}$ of some most similar citations; MMI uses MetaMap to extract biomedical concepts from title and abstract, which are then mapped to MHs. These two sets of MHs are combined, ranked and recommended to the NLM curators after some post-processing, such as applying indexing rules.

Since 2013, many more advanced machine learning based methods have been proposed to tackle the problem of large-scale MeSH indexing, which is greatly facilitated by BioASQ challenges (2013-2019) that provide a practical and realistic benchmark for performance comparison (Tsatsaronis et al., 2015). Based on the machine learning techniques used, these automatic methods can be divided into three categories. (i) Binary relevance (BR); The best system in BioASQ 2013 developed by Tsoumakas et al. (2013), MetaLabeler, belongs to this category, where a linear SVM classifier is trained for each MH independently. Given a test citation, the candidate $\mathrm{MHs}$ are ranked according to the prediction score of each MH classifier. (ii) Learning to rank (LTR); MeSH Now (Mao and Lu, 2017), MeSHLabeler (Liu et al., 2015) and DeepMeSH (Peng et al., 2016) are three representative methods in this category. The main idea is to model MeSH indexing as a problem of ranking multiple MHs, where top ranked MHs are recommended as true labels. LTR has been successfully applied in the field of information retrieval, such as web searching. In the case of MeSH indexing, multiple evidence generated from different text representations and machine learning models are effectively integrated by LTR to improve the performance. Note that MeSHLabeler achieved the first place in BioASQ 2014 and 2015, while DeepMeSH achieved the first place in BioASQ 2016, 2017 and 2019. (iii) Deep Learning; AttentionMeSH (Jin et al., 2018) and MeSHProbeNet (Xun et al., 2019) are two recent deep learning based methods, which both use deep recursive neural network (RNN) and attention mechanism. Specifically, MeSHProebNet achieved the first place in BioASQ 2018 and the second place in BioASQ 2019, while AttentionMeSH achieved the third place in BioASQ 2018. 


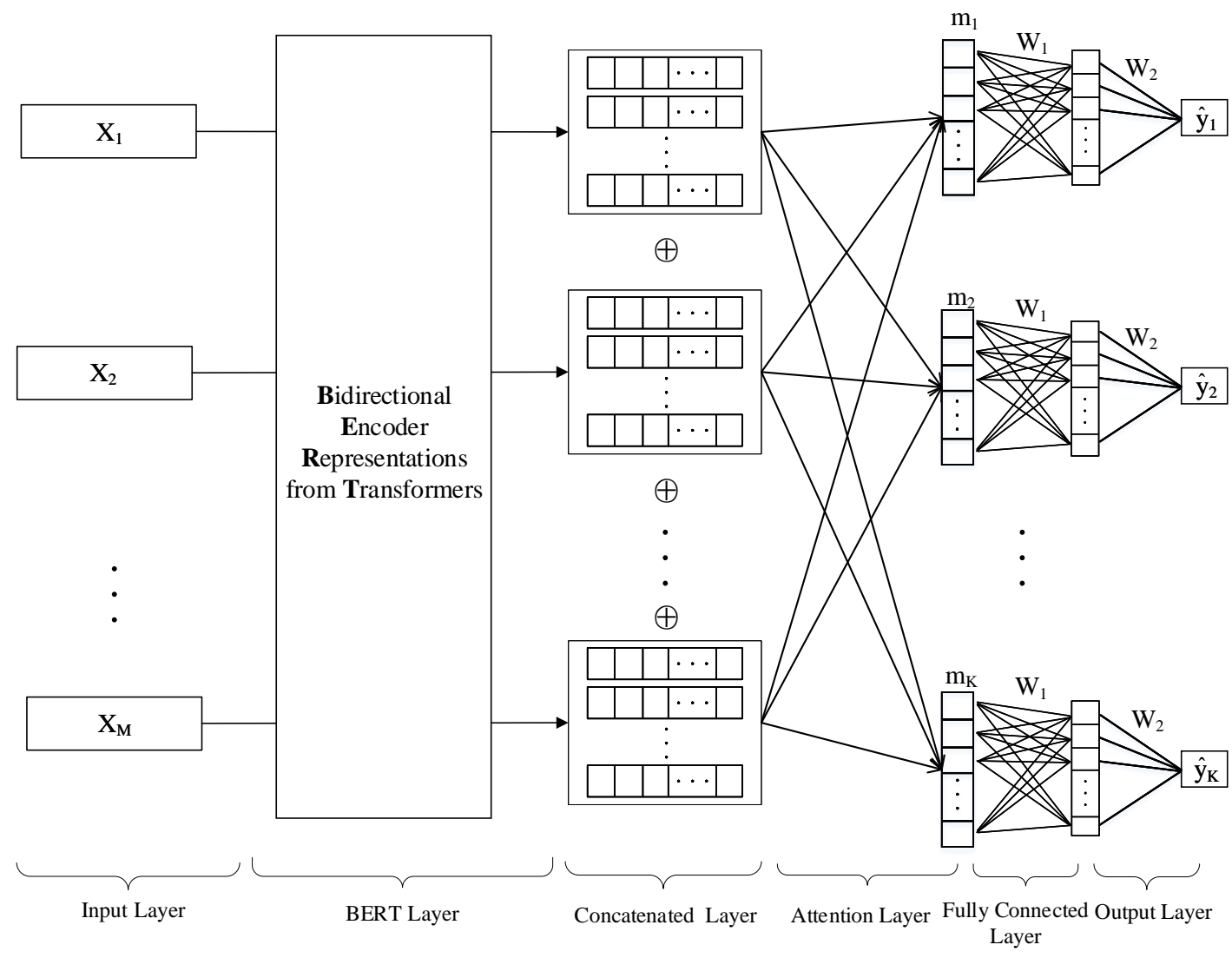

Fig. 1. The architecture of BERTMeSH

Note that all these methods used title and abstract only, which cannot take advantage of rich information in full text. In addition, although AttentionMeSH and MeSHProbNet are two deep learning based methods, they cannot enjoy the recent progress in pre-training in natural language processing (NLP).

\subsection{BERT: Bidirectional Encoder Representations from Transformers}

Deep learning models for NLP tasks used raw texts as inputs to capture rich semantic context information. Previously deep learning methods for NLP tasks usually used word embeddings (Mikolov et al., 2013) pre-trained on a large corpus to covert words to their corresponding dense semantic vectors, such as Word2Vec (Mikolov et al., 2013) and GloVe (Pennington et al., 2014). In spite of some successful applications in NLP including MeSH indexing (Peng et al., 2016), this type of word embedding uses an identical vector (representation) for the same word in different sentences, which cannot model the local context very well. Some pre-trained contextual text representations were then developed for replacing the single word embedding, such as ELMo (Embeddings from Language Models) (Peters et al., 2018) and BERT (Bidirectional Encoder Representation from Transformers) (Devlin et al., 2019). Different from previous language representation models like ELMo, BERT considers both left and right context of text when learning the language representation. The pre-trained BERT model has found many successful applications in NLP. Given the excitement about BERT, it was noted that it should be applied to biomedical NLP (Burns et al., 2019). Most recently, based on BERT, Lee et al. (2020) trained a biomedical domain specific language model BioBERT using biomedical text corpus such MEDLINE and PMC. They found that BioBERT improved the performance of several typical biomedical text mining tasks, such as biomedical name entity recognition, relation extraction and question answering. In this work, we used BioBERT for text representation, which greatly improves the performance of large-scale MeSH indexing.

\section{Methods: BERTMeSH}

\subsection{Overview}

Fig. 1 shows the architecture of BERTMeSH. For each biomedical article, we use the raw text from title and abstract and the $M-1$ longest sections from body text as our inputs. A pre-trained BERT layer (Devlin et al., 2019) is employed to obtain deep contextual representation of each word For reducing the scale (the number of parameters) of our model, we use an identical BERT layer for all sections. Then we concatenate the outputs of all sections after the BERT layer as the representation of a given article. Following AttentionCNN, a deep component model of FullMeSH (Dai et al., 2020), we use a multi-label attention over the gained representation to capture the most relevant parts to each label, resulting in a different representation to each label. Finally, we use fully connected layers with sharing weights to obtain the predicted score to each label. To take advantage of both full text from PMC and a large amount of labeled citations from MEDLINE (which have title and abstract only), we use a transfer learning strategy. Specifically, BERTMeSH is pre-trained with millions of MEDLINE citations first, and then fine tuned with PMC full text data. 


\subsection{Input Layer}

For each sample, we use the raw text of $M$ sections as our inputs, including the title and abstract section and the $M-1$ longest sections from the body text. The input $\boldsymbol{X}_{k}$ of the $k$-th section for a given sample is as follows:

$$
\boldsymbol{X}_{k}=\left(x_{k, 1}, x_{k, 2}, \ldots, x_{k, i}, \ldots, x_{k, T}\right)
$$

where the $x_{k, i}$ is the $i$-th word in the $k$-th section and $T$ is the text length of each section.

\subsection{BERT Layer}

We use BioBERT (Lee et al., 2020) as our text representation model. BioBERT fine-tunes pre-trained BERT-base on MEDLINE and PMC corpus. The output $\boldsymbol{H}_{k}$ of the $k$-th section is as follows:

$$
\boldsymbol{H}_{k}=\operatorname{BERT}\left(\theta_{\mathrm{BERT}}, \boldsymbol{X}_{j}\right)=\left(h_{k, 1}, h_{k, 2}, \ldots, h_{k, T}\right)
$$

where $\boldsymbol{H}_{k} \in \mathbb{R}^{T \times n}, n$ is the hidden size of BERT, $\theta_{\mathrm{BERT}}$ is the weight parameter of BERT Layer, and $h_{k, i} \in \mathbb{R}^{n}$ is the representation of the $i$-th word in the $k$-th section.

\subsection{Concatenated Layer}

For a given biomedical article, we concatenate $M$ outputs of BERT layer over all $M$ sections as follows:

$$
\boldsymbol{H}=\boldsymbol{H}_{1} \oplus \boldsymbol{H}_{2} \oplus \ldots \oplus \boldsymbol{H}_{M}
$$

where $\boldsymbol{H}\left(\in \mathbb{R}^{M T \times n}\right)$ is the concatenated output, and $M T=M \times$ $T$. We denote $\mathbf{h}_{i}\left(\in \mathbb{R}^{n}\right)$ as the representation of the $i$-th word in the concatenated output $\boldsymbol{H}$.

\subsection{Multi-label Attention}

We use a multi-label attention to capture the most relevant part of text to each label to have different representations for each label. We use different attention parameters for each label. For the $j$ th label, the attention we use is as follows:

$$
\mathbf{m}_{j}=\sum_{i=1}^{M T} \alpha_{i j} \mathbf{h}_{i}, \quad \alpha_{i j}=\frac{e^{\mathbf{h}_{i} \mathbf{w}_{j}}}{\sum_{t=1}^{M T} e^{\mathbf{h}_{t} \mathbf{w}_{j}}}
$$

where $\mathbf{w}_{j}$ is the attention weight for the $j$-th label and $\mathbf{m}_{j}$ is the attention output for the $j$-th label.

\subsection{Fully Connected Layer and Output Layer}

BERTMeSH has one fully connected layer and one output layer. We set up that the fully connected layer and the output layer share the same paramete values for all labels, to emphasize the differences of attention among all labels and reduce the number of parameters. Finally, predicted probability $\hat{y}_{j}$ for the $j$-th label can be computed as follows:

$$
\hat{y}_{j}=\sigma\left(\mathbf{W}_{2} f\left(\mathbf{W}_{1} \mathbf{m}_{j}+\mathbf{b}_{1}\right)+b_{2}\right)
$$

where $\mathbf{W}_{1}\left(\in \mathbb{R}^{c \times n}\right)$ and $\mathbf{W}_{2}\left(\in \mathbb{R}^{c}\right)$ are parameters of the fully connected layer and output layer, respectively, and $\mathbf{b}_{1}\left(\in \mathbb{R}^{\hat{n}}\right)$ and $b_{2}(\in \mathbb{R})$ are bias terms, and $f$ is a non-linear (activation) function.

\subsection{Loss Function}

BERTMeSH uses the binary cross-entropy loss, as the loss function, which is given as follows:

$$
J(\theta)=-\frac{1}{N K} \sum_{i=1}^{N} \sum_{j=1}^{K} y_{i j} \log \left(\hat{y}_{i j}\right)+\left(1-y_{i j}\right) \log \left(1-\hat{y}_{i j}\right), \quad \text { (6) }
$$

where $N$ is the number of samples, $K$ is the number of labels, $\hat{y}_{i j} \in[0,1]$ and $y_{i j} \in\{0,1\}$ are the predicted probability and true value, respectively, for the $i$-th sample and the $j$-th label.

\subsection{Threshold for Each Label}

After training, we compute the optimal threshold for each label over a threshold validation set, following (Pillai et al., 2013). We then select MHs with higher scores than the threshold as the final recommended MHs.

\subsection{Pre-training with MEDLINE citations}

Citations in PMC have full text, which has more useful information than title and abstract only. While the number of citations in MEDLINE with only title and abstract, is much larger than the number of citations in PMC. To improve the performance, we use transfer learning, to take advantage of both PMC and MEDLINE citations. Thus we first train BERTMeSH on MEDLINE citations with only title and abstract, and then fine-tunes this model with PMC citations.

\section{Results}

\subsection{Data collection}

We downloaded the whole PMC open access subset (by Oct. 2019) ${ }^{5}$ and obtained 3,221,713 citations. We also downloaded the whole MEDLINE collections (by Oct. 2019) ${ }^{6}$ and obtained 16,677,027 citations with abstract. For reducing the bias, we focused on manually indexed citations (not annotated by a "curated" or "auto" modes in MEDLINE) only in our work, which also have full text in PMC open access subset. Then we obtained a set of 1,495,063 PMC articles. Out of all these PMC articles, we used the latest 20,000 articles as the test set, another latest 200,000 articles except the test set as the threshold validation data set for all $\mathrm{MHs}$, and the remaining $1.27 \mathrm{M}$ articles as the training set. We used the latest $3,000,000$ MEDLINE citations as our MEDLINE pre-training dataset, which were annotated before the earliest date of the threshold validation data set. This means that this 3M MEDLINE citations have no overlap with our threshold validation and test datasets. Following the pattern matching method in FullMeSH, in addition to Title and Abstract, we extracted the 4 pre-defined sections from the test data, Introduction, Methods and Materials, Result and Experiment, Conclusion and Summary. Out of all 20,000 test articles, $13,641(67.3 \%)$ articles had all these 5 sections, and the remaining 6,359 articles $(32.7 \%)$ lost one or more sections in full text, where we call these two subsets the complete subset and incomplete subset, respectively.

\subsection{Experimental Settings}

As BERT Layer (with 12 transformer layer), we used BioBERT (Lee et al., 2020), which fine-tuned pre-trained BERT-base (Devlin et al., 2019) on MEDLINE and PMC open access subset with $n=768$ and $T=512$ We used the Adam optimizer (Kingma and Ba, 2014). Also we used a dropout with the drop rate of 0.5 and early stopping to avoid overfitting. We used five sections for training by PMC articles, including title and abstract, and the four longest sections $(M=5)$. There are six variant

\footnotetext{
5 ftp://ftp.ncbi.nlm.nih.gov/pub/pmc

${ }^{6} \mathrm{ftp} / / / \mathrm{ftp}$. ncbi.nlm.nih.gov/pubmed/baseline
} 
Table 1. Performance comparison of BERTMeSH and DeepMeSH by using title and abstract only.

\begin{tabular}{ccccccccccccc}
\hline Method & Training & pre-trained & Full Text & MiF & MiP & MiR & MaF & MaP & MaR & EBF & EBP & EBR \\
\hline DeepMeSH & PMC & $\times$ & $\times$ & 0.639 & 0.669 & 0.612 & 0.495 & 0.633 & 0.502 & 0.631 & 0.667 & 0.627 \\
DeepMeSH & MEDLINE & $\times$ & $\times$ & 0.653 & 0.690 & 0.621 & 0.540 & 0.657 & 0.545 & 0.646 & 0.687 & 0.638 \\
BERTMeSH w.o. BERT Layer & MEDLINE & $\times$ & $\times$ & 0.662 & 0.695 & 0.631 & 0.525 & 0.668 & 0.526 & 0.652 & 0.700 & 0.643 \\
BERTMeSH & PMC & $\times$ & $\times$ & 0.667 & 0.696 & 0.640 & 0.512 & 0.663 & 0.517 & 0.657 & 0.700 & 0.650 \\
BERTMeSH & MEDLINE & $\times$ & $\times$ & $\mathbf{0 . 6 7 8}$ & $\mathbf{0 . 7 0 5}$ & $\mathbf{0 . 6 5 3}$ & $\mathbf{0 . 5 5 0}$ & $\mathbf{0 . 6 7 8}$ & $\mathbf{0 . 5 5 5}$ & $\mathbf{0 . 6 7 0}$ & $\mathbf{0 . 7 1 1}$ & $\mathbf{0 . 6 6 3}$ \\
& & & & & & & & & & & &
\end{tabular}

Table 2. Performance comparison of BERTMeSH and FullMeSH by using full text.

\begin{tabular}{ccccccccccccc}
\hline Method & Training & pre-trained & Full Text & MiF & MiP & MiR & MaF & MaP & MaR & EBF & EBP & EBR \\
\hline FullMeSH & PMC & $\times$ & $\checkmark$ & 0.651 & 0.683 & 0.623 & 0.512 & 0.647 & 0.516 & 0.643 & 0.680 & 0.639 \\
BERTMeSH & PMC & $\times$ & $\checkmark$ & 0.684 & 0.711 & 0.660 & 0.526 & 0.666 & 0.532 & 0.674 & 0.715 & 0.667 \\
BERTMeSH & PMC & PMC & $\checkmark$ & 0.685 & 0.713 & 0.659 & 0.528 & 0.670 & 0.533 & 0.675 & 0.717 & 0.667 \\
BERTMeSH & PMC & MEDLINE & $\checkmark$ & $\mathbf{0 . 6 9 2}$ & $\mathbf{0 . 7 1 9}$ & $\mathbf{0 . 6 6 8}$ & $\mathbf{0 . 5 6 2}$ & $\mathbf{0 . 6 8 3}$ & $\mathbf{0 . 5 6 8}$ & $\mathbf{0 . 6 8 3}$ & $\mathbf{0 . 7 2 4}$ & $\mathbf{0 . 6 7 6}$
\end{tabular}

of BERTMeSH. First three variants use title and abstract only. They use PMC data, MEDLINE citations, and MEDLINE citations with Word2Vec and RNN instead of BERT layer, respectively. The other three variants use full text, and in practice, use PMC data only without pre-training, PMC data with pre-trained network by PMC abstracts, and PMC data with pre-trained network by MEDLINE citations.

Since the implementation of MeSHProbNet and AttentionMeSH for large-scale MeSH indexing is not available, we used DeepMeSH and FullMeSH as two competing methods, which are the state-of-the-art MeSH indexing methods using abstract and full text, respectively. Following the original paper (Dai et al., 2020), we implemented DeepMeSH and FullMeSH. Specifically, the same 20,000 latest PMC articles were used as the test set. Other 40,000 latest PMC articles were extracted, and half of the 40,000 articles were randomly chosen to train the ranking model, and the rest were used to train the model to predict the number of MHs annotated for a given article. Finally the remaining 1.4M articles were used as the training data. Note that FullMeSH used the full text of PMC, while DeepMeSH used the title and abstract only. In addition, we also checked the performance of DeepMeSH using the whole MEDLINE collection as the training data, after removing the above 60,000 citations.

\subsection{Performance evaluation measures}

Let $K$ be the size of all labels (MHs), and $N$ be the number of instances (citations). Let $y_{i}$ and $\hat{y}_{i} \in\{0,1\}^{K}$ be the true and predicted labels for instance $i$, respectively. For performance evaluation, we used the three groups of most common metrics: Micro (precision (MiP), recall (MiR) and F-Measure (MiF)), Macro (precision (MaP), recall (MaP) and F-Measure $(\mathrm{MaF})$ ) and Example Based (precision (EBP), recall (EBR) and F-Measure $(\mathrm{EBF})$ ), which are defined as follows:

$$
\begin{gathered}
\mathrm{MiP}=\frac{\sum_{k=1}^{K} \sum_{i=1}^{N} y_{i}^{k} \cdot \hat{y}_{i}^{k}}{\sum_{k=1}^{K} \sum_{i=1}^{N} \hat{y}_{i}^{k}} \\
\mathrm{MiR}=\frac{\sum_{k=1}^{K} \sum_{i=1}^{N} y_{i}^{k} \cdot \hat{y}_{i}^{k}}{\sum_{k=1}^{K} \sum_{i=1}^{N} y_{i}^{k}} \\
\mathrm{MiF}=\frac{2 \cdot \mathrm{MiP} \cdot \mathrm{MiR}}{\mathrm{MiP}+\mathrm{MiR}} \\
\mathrm{MaP}_{k}=\frac{\sum_{i=1}^{N} y_{i}^{k} \cdot \hat{y}_{i}^{k}}{\sum_{i=1}^{N} \hat{y}_{i}^{k}} \\
\mathrm{MaR}_{k}=\frac{\sum_{i=1}^{N} y_{i}^{k} \cdot \hat{y}_{i}^{k}}{\sum_{i=1}^{N} y_{i}^{k}}
\end{gathered}
$$

$$
\begin{gathered}
\mathrm{MaF}=\frac{1}{K} \sum_{k=1}^{K} \frac{2 \cdot \mathrm{MaP}_{k} \cdot \mathrm{MaR}_{k}}{\mathrm{MaP}_{k}+\mathrm{MaR}_{k}} . \\
\mathrm{EBP}_{i}=\frac{\sum_{k=1}^{K} y_{i}^{k} \cdot \hat{y}_{i}^{k}}{\sum_{k=1}^{K} \cdot \hat{y}_{i}^{k}} \\
\mathrm{EBR}_{i}=\frac{\sum_{k=1}^{K} y_{i}^{k} \cdot \hat{y}_{i}^{k}}{\sum_{k=1}^{K} y_{i}^{k}} . \\
\mathrm{EBF}=\frac{1}{N} \sum_{i=1}^{N} \frac{2 \cdot \mathrm{EBP}_{i} \cdot \mathrm{EBR}_{i}}{\mathrm{EBP}_{i}+\mathrm{EBR}_{i}}
\end{gathered}
$$

\subsection{Experimental results}

By using the 20,000 benchmark test articles, we compared the performance of BERTMeSH with DeepMeSH using title and abstract only, and then with FullMesH using full text. We mainly focused on MiF, the primary evaluation metric in the BioASQ challenge.

4.4.1 Performance comparison with title and abstract only

Table 1 shows the performance comparison result of two settings of DeepMeSH (trained with PMC and trained with MEDLINE) and three settings of BERTMeSH (trained with PMC, trained with MEDLINE, and trained with MEDLINE using Word2Vec and RNN instead of BERT Layer). In Table 1, BERTMeSH outperformed DeepMeSH under al settings. BERTMeSH trained on MEDLINE achieved the best performance among the five settings. Specifically, BERTMeSH trained on MEDLINE achieved MiF of 0.678 , being followed by BERTMeSH trained with PMC (0.667), BERTMeSH trained with MEDLINE using Word2Vec and RNN instead of BERT layer (0.662), DeepMeSH trained with MEDLINE (0.653), and DeepMeSH trained with PMC (0.639). We can see that with more training data (using MEDLINE instead of PMC), the performance of both DeepMeSH and BERTMesH was improved significantly in all three F-measures. For example, the MaF of DeepMeSH increased from 0.495 to 0.540 . Another finding is that, without BERT layer, the performance of BERTMeSH trained with MEDLINE is even worse than BERTMeSH trained with PMC. For example, BERTMeSH trained with MEDLINE without BERT layer achieved MiF of 0.662, where BERTMeSH trained with PMC achieved MiF of 0.667. This highlights the power of deep contextual representation for improving the performance of $\mathrm{MeSH}$ indexing. 
Table 3. Performance over full text with full sections.

\begin{tabular}{ccccccccccccc}
\hline Method & Training & pre-trained & Full Text & MiF & MiP & MiR & MaF & MaP & MaR & EBF & EBP & EBR \\
\hline DeepMeSH & PMC & $\times$ & $\times$ & 0.646 & 0.679 & 0.616 & 0.501 & 0.637 & 0.507 & 0.639 & 0.677 & 0.630 \\
FullMeSH & PMC & $\times$ & $\checkmark$ & 0.661 & 0.705 & 0.623 & 0.516 & 0.662 & 0.516 & 0.654 & 0.703 & 0.637 \\
BERTMeSH & PMC & $\times$ & $\checkmark$ & 0.691 & 0.714 & 0.669 & 0.533 & 0.666 & 0.541 & 0.681 & 0.718 & 0.676 \\
BERTMeSH & PMC & MEDLINE & $\checkmark$ & $\mathbf{0 . 6 9 8}$ & $\mathbf{0 . 7 2 1}$ & $\mathbf{0 . 6 7 6}$ & $\mathbf{0 . 5 6 3}$ & $\mathbf{0 . 6 8 0}$ & $\mathbf{0 . 5 7 2}$ & $\mathbf{0 . 6 8 8}$ & $\mathbf{0 . 7 2 5}$ & $\mathbf{0 . 6 8 4}$
\end{tabular}

Table 4. Performance over full text with missing sections.

\begin{tabular}{ccccccccccccc}
\hline Method & Training & pre-trained & Full Text & MiF & MiP & MiR & MaF & MaP & MaR & EBF & EBP & EBR \\
\hline DeepMeSH & PMC & $\times$ & $\times$ & 0.621 & 0.644 & 0.601 & 0.494 & 0.613 & 0.506 & 0.616 & 0.647 & 0.621 \\
FullMeSH & PMC & $\times$ & $\checkmark$ & 0.626 & 0.628 & 0.625 & 0.512 & 0.604 & 0.531 & 0.620 & 0.633 & 0.645 \\
BERTMeSH & PMC & $\times$ & $\checkmark$ & 0.666 & 0.700 & 0.634 & 0.528 & 0.656 & 0.535 & 0.659 & 0.709 & 0.648 \\
BERTMeSH & PMC & MEDLINE & $\checkmark$ & $\mathbf{0 . 6 7 8}$ & $\mathbf{0 . 7 1 4}$ & $\mathbf{0 . 6 4 6}$ & $\mathbf{0 . 5 5 9}$ & $\mathbf{0 . 6 7 7}$ & $\mathbf{0 . 5 6 4}$ & $\mathbf{0 . 6 7 2}$ & $\mathbf{0 . 7 2 2}$ & $\mathbf{0 . 6 6 1}$ \\
\hline
\end{tabular}

Table 5. Statistical significance test by bootstrapping

\begin{tabular}{|c|c|c|c|c|c|c|c|c|c|c|c|c|}
\hline Method & Training & pre-trained & Full Text & $\mathrm{MiF}$ & $\mathrm{MiP}$ & $\mathrm{MiR}$ & $\mathrm{MaF}$ & $\mathrm{MaP}$ & $\mathrm{MaR}$ & EBF & EBP & EBR \\
\hline \multirow[t]{2}{*}{ DeepMeSH } & MEDLINE & $x$ & $x$ & 0.653 & 0.690 & 0.621 & 0.539 & 0.656 & 0.549 & 0.646 & 0.687 & 0.638 \\
\hline & & & & $1.91 \mathrm{e}-169$ & 7.66e-144 & $3.56 \mathrm{e}-168$ & $3.92 \mathrm{e}-109$ & $3.62 \mathrm{e}-110$ & $2.96 \mathrm{e}-110$ & $3.20 \mathrm{e}-164$ & $1.65 \mathrm{e}-150$ & $4.04 \mathrm{e}-159$ \\
\hline \multirow[t]{2}{*}{ FullMeSH } & PMC & $x$ & $\checkmark$ & 0.651 & 0.683 & 0.623 & 0.513 & 0.645 & 0.522 & 0.643 & 0.680 & 0.639 \\
\hline & & & & $1.48 \mathrm{e}-176$ & $4.35 \mathrm{e}-16$ & $2.41 \mathrm{e}-169$ & $2.43 \mathrm{e}-144$ & $1.38 \mathrm{e}-124$ & $3.92 \mathrm{e}-143$ & $1.32 \mathrm{e}-169$ & $5.14 \mathrm{e}-160$ & $7.84 \mathrm{e}-158$ \\
\hline \multirow[t]{2}{*}{ BERTMeSH } & MEDLINE & $x$ & $x$ & 0.678 & 0.705 & 0.653 & 0.551 & 0.677 & 0.562 & 0.670 & 0.711 & 0.663 \\
\hline & & & & $3.58 \mathrm{e}-142$ & $7.23 \mathrm{e}-129$ & $1.83 \mathrm{e}-136$ & $1.72 \mathrm{e}-97$ & $1.06 \mathrm{e}-51$ & $2.26 \mathrm{e}-98$ & $9.39 \mathrm{e}-136$ & $8.18 \mathrm{e}-127$ & $1.44 \mathrm{e}-128$ \\
\hline BERTMeSH & PMC & MEDLINE & $\checkmark$ & 0.692 & 0.719 & 0.668 & 0.561 & 0.681 & 0.572 & 0.683 & 0.724 & 0.676 \\
\hline
\end{tabular}

\subsubsection{Performance comparison with full text}

Table 2 shows the performance comparison result of FullMeSH and three settings of BERTMeSH (pre-trained with PMC, pre-trained MEDLINE and without pre-training). In Table 2, BERTMeSH outperformed FullMeSH under all settings, and BERTMeSH pre-trained with MEDLINE achieved the best performance. Specifically, BERTMeSH pre-trained with MEDLINE achieved MiF of 0.692, which is 6.3\% higher than FullMeSH (0.651). Even without pre-training, the performance of BERTMeSH reached $\mathrm{MiF}$ of 0.684 , which is still much higher than the performance of FullMeSH. Note that if BERTMeSH is pre-trained with PMC itself, the performance increase is slight. For example, MiF of BERTMeSH increases from 0.684 to 0.685 .

4.4.3 Performance comparison with missing sections: validation on robustness

In Section 4.1, we divided the whole test data into two subsets: complete subset and incomplete subset. Again the complete subset consists of 13,46 $(67.3 \%)$ test articles, each with all five sections, and the incomplete subset consists of 6,539 (32.7\%) test article, each losing at least one section. In Table 2, BERTMeSH pre-trained with PMC had only little improvement, and then we examined the performance of three methods: BERTMeSH without pre-training, BERTMeSH pre-trained with MEDLINE, and FullMeSH. In addition, DeepMeSH using PMC abstract was also examined as a baseline. Tables 3 and 4 show the performance results of all competing methods over the complete and incomplete subsets, respectively. BERTMeSH pre-trained with MEDLINE achieved the best performance in both cases, being followed by BERTMeSH without pre-training, FullMeSH and DeepMeSH. For example, over the complete subset, BERTMeSH pre-trained with MEDLINE achieved MiF of 0.698, which was followed by BERTMeSH without pre-training (0.691), FullMeSH (0.661) and DeepMeSH (0.646). Another point of note is that
BERTMeSH is more robust than FullMeSH regarding missing sections, which can be seen from two viewpoints: 1) with missing sections, the MiF of BERTMeSH pre-trained with MEDLINE decreased 2.9\%, i.e. from 0.698 to 0.678 , and also that of BERTMeSH without pre-training decreased $3.6 \%$, i.e. from 0.691 to 0.666 . However, the decrease of FullMeSH was $5.3 \%$, i.e. from 0.661 to 0.626 . 2) over the complete subset, the MiF of BERTMeSH was $5.6 \%$ higher than that of FullMeSH, while over the incomplete subset, the MiF of BERTMeSH was $8.3 \%$ higher than that of FullMeSH. All these suggest BERTMeSH is more robust than FullMeSH with respect to the organization of sections in full text.

\subsubsection{Statistical performance superiority confirmation}

By using the test set of 20,000 articles, we repeated boostrap with replacement 100 times, to generate 100 data sets. We then conducted paired $t$-test over 100 trials to examine the statistical significance on performance improvement between BERTMeSH and two state-of-the-art competing methods (DeepMeSH and FullMeSH). For BERTMeSH, we consider the two best settings: BERTMeSH with MEDLINE pre-training and BERTMeSH trained with MEDLINE. For DeepMeSH, hereafter we consider its best setting, which was trained with MEDLINE abstracts. Table 5 reports the predictive and statistical results of BERTMeSH, being compared with DeepMeSH and FullMeSH. In this table, below the performance values, the corresponding $p$-values are shown. Regarding the performance, BERTMeSH with PMC full text pre-trained with MEDLINE achieved the highest $\mathrm{MiF}$ of 0.692 , being followed by BERTMeSH with MEDLINE abstract (0.678), DeepMeSH (0.653) and FullMeSH (0.651). Also from the $p$-values, which are far smaller than the regular statistical significance level, such as 0.05 , the performance improvement by BERTMeSH was statistically significant. Overall the experimental results can be summarized into the following three points: 1) BERTMeSH outperformed other baselines for large-scale $\mathrm{MeSH}$ indexing, being 

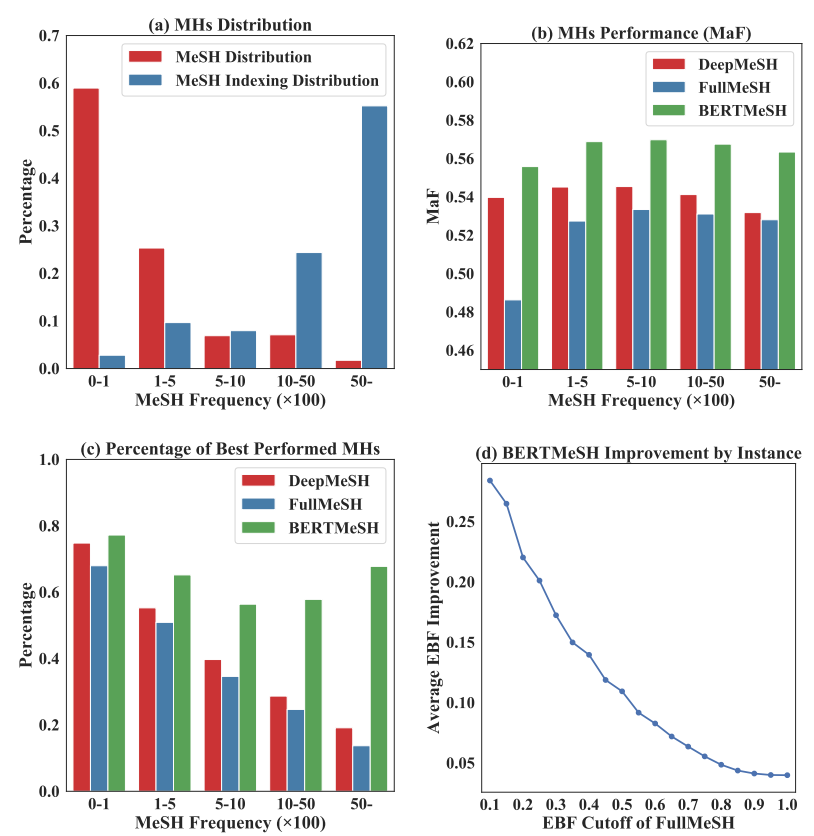

Fig. 2. Performance comparison of DeepMeSH, FullMeSH and BERTMeSH

statistically significant; 2) the performance of BERTMeSH was improved with full text; and 3) BERTMeSH shows the robustness over FullMeSH regarding missing sections in full text.

\subsection{Result Analysis}

4.5.1 Performance comparison of three methods under different frequencies of MHs and articles

We divided MHs into five groups by the number of occurrences in training set: $[0,100),[100,500),[500,1,000),[1,000,5,000)$ and $[5000$,$) . Fig. 2(a)$ shows the distributions of MHs and MHs indexing, when we divided training set into the above five groups. Fig. 2(b) shows the performance (average MaF) of BERTMeSH, FullMeSH and DeepMeSH, in each of the above five groups of MHs. For all groups, BERTMeSH achieved the best performance, being followed by DeepMeSH and FullMeSH. This highlights the advantage of BERTMeSH over DeepMeSH and FullMeSH, regardless of the frequency of MHs. Fig. 2(c) shows the distributions of the three methods regarding the best predictive methods (allowing ties) for each $\mathrm{MH}$, for each of the five frequency groups. From Figs. 2(b) and 2(c), BERTMeSH outperformed DeepMeSH and FullMeSH, particularly more significantly for higher frequency groups. Another point of note is that for the most infrequent group, MaF of FullMeSH is much worse than DeepMeSH, while for the most frequent group, MaF of FullMeSH is only slight lower than DeepMeSH. This might be because: the size of the training set (MEDLINE) of DeepMeSH (which uses title and abstract only) is much larger than that (PMC) of FullMeSH (which allows to use all full text). That is, DeepMeSH would have used much more positive samples than FullMeSH. This might be very helpful for predicting infrequent MHs.

Furthermore, we compared the performance of BERTMeSH with FullMeSH with respect to instances (articles). We changed the size of test data, using EBF of each article by FullMeSH as the cut-off value, and then checked the performance improvement in the average EBF by changing the cut-off value. Fig. 2(d) shows the average EBF improvement of BERTMeSH over FullMeSH. From this figure, the improvement of BERTMeSH becomes larger, as EBF of FullMeSH became smaller,
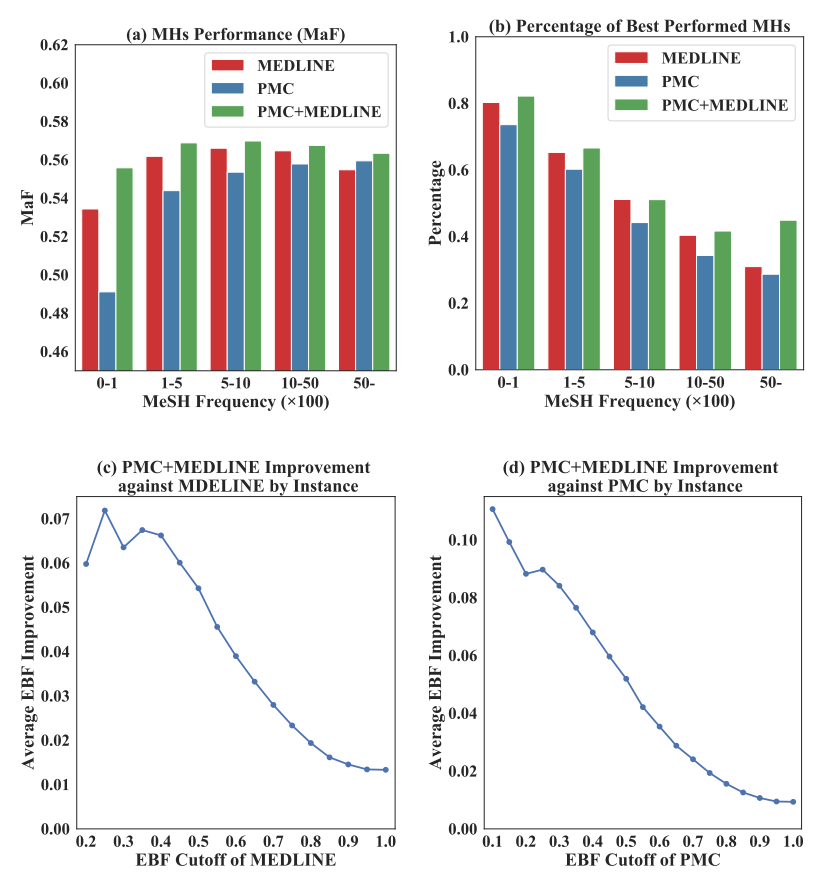

Fig. 3. Performance comparison of BERTMeSH trained on MEDLINE, PMC and PMC with a pre-training over MEDLINE.

meaning that the article for which prediction is hard by FullMeSH can be better predicted by BERTMeSH.

\subsubsection{Performance comparison of different data usage for BERTMeSH under different frequencies of MHs and articles}

We consider three data usages for BERTMeSH: 1) MEDLINE BERTMeSH trained on only title and abstract in MEDLINE, 2) PMC: BERTMeSH trained on full text in PMC and 3) PMC+MEDLINE: BERTMeSH trained on full text in PMC with pre-training on MEDLINE. Fig. 3(a) shows the performance (MaF) of these three cases. First, focusing on MEDLINE and PMC only, MEDLINE outperformed PMC for all groups, except the most frequent group, particularly the performance advantage being wider for the groups with lower frequencies. This highlights the advantage of using MEDLINE for predicting less frequent MHs, which would be a large portion of the data set. On the other hand, PMC made better prediction on the high frequency group, although PMC had less training data (\#articles would be smaller). For these most frequent MHs, which are usually important and general biomedical concepts, related information may be described in the full text other than abstract. In this case, PMC would be more advantageous than MEDLINE. Also Fig. 3(b) shows the distributions of the three data usages regarding the best predictive usage for each $\mathrm{MH}$, for each of the five frequency groups. From Figs. 3(a) and 3(b), PMC+MEDLINE always outperformed the other two usages. This demonstrates that PMC+MEDLINE can enjoy the advantages of both pre-training by MEDLINE and training by full text of PMC.

Furthermore, we again changed the size of test data, using the EBF of each article by MEDLINE (or PMC) as the cut-off value, and examined the performance improvement by PMC+MEDLINE. Figs. 3(c) and 3(d) show the average EBF improvement of PMC+MEDLINE over MEDLINE and PMC, respectively. The improvement of PMC+MEDLINE becomes larger, as the EBF of MEDLINE (or PMC) became smaller, meaning that the article for which prediction is hard by MEDLINE (or PMC) only can be better predicted by PMC+MEDLINE. 
Table 6. Performance (F1-score) comparison over Check Tags in test set (number of occurrence more than 150).

\begin{tabular}{ccccc}
\hline Check Tags & \#N & MEDLINE & PMC & PMC+MEDLINE \\
\hline Humans & 14,121 & 0.955 & 0.958 & $\mathbf{0 . 9 6 0}$ \\
Female & 8,336 & 0.873 & 0.897 & $\mathbf{0 . 8 9 8}$ \\
Male & 7,842 & 0.863 & 0.885 & $\mathbf{0 . 8 8 6}$ \\
Animals & 5,810 & 0.916 & 0.922 & $\mathbf{0 . 9 2 5}$ \\
Middle Aged & 4,520 & 0.841 & 0.885 & $\mathbf{0 . 8 8 8}$ \\
Adult & 4,288 & 0.788 & 0.842 & $\mathbf{0 . 8 4 8}$ \\
Aged & 3,145 & 0.765 & 0.836 & $\mathbf{0 . 8 3 7}$ \\
Young Adult & 1,897 & 0.652 & $\mathbf{0 . 7 4 8}$ & $\mathbf{0 . 7 4 8}$ \\
Mice & 1,895 & 0.808 & $\mathbf{0 . 8 2 9}$ & 0.828 \\
Adolescent & 1,601 & 0.661 & 0.775 & $\mathbf{0 . 7 7 8}$ \\
Aged, 80 and over & 1,189 & 0.528 & 0.734 & $\mathbf{0 . 7 4 2}$ \\
Child & 1006 & 0.747 & $\mathbf{0 . 8 3 2}$ & 0.831 \\
Child, Preschool & 703 & 0.722 & $\mathbf{0 . 8 0 8}$ & 0.803 \\
Rats & 647 & 0.804 & $\mathbf{0 . 8 1 8}$ & 0.801 \\
Infant & 618 & 0.711 & $\mathbf{0 . 8 0 6}$ & 0.801 \\
Pregnancy & 604 & 0.887 & 0.886 & $\mathbf{0 . 8 8 9}$ \\
Infant, Newborn & 442 & 0.673 & 0.720 & $\mathbf{0 . 7 2 1}$ \\
Cattle & 212 & 0.872 & 0.872 & $\mathbf{0 . 8 7 6}$ \\
Dogs & 197 & 0.890 & 0.920 & $\mathbf{0 . 9 2 6}$ \\
\hline Average & - & 0.787 & $\mathbf{0 . 8 4 1}$ & $\mathbf{0 . 8 4 1}$ \\
\hline
\end{tabular}

\subsubsection{Performance comparison of data usages on high frequency MHs} by using Check Tags

Check Tags are a set of most frequent MHs, such as human, male, female and animal, meaning that Check Tags are likely to be mentioned in each article. In Section 4.5.2, we found that PMC (trained by full text) performed well for the most frequent MHs. We further check a similar but different setting of BERTMeSH by using Check Tags. We found 19 Check Tags that occur more than 150 times in our test set. Table 6 shows the F1score of BERTMeSH by using three different data usages: MEDLINE PMC and PMC+MEDLINE (which are the same as mentioned in Section 4.5.2) on the 19 Check Tags. In this table, PMC+MEDLINE achieved the highest F1-score in 14 out of 19 Check Tags, being followed by PMC, which achieved the highest in 6 Check Tags. Also both PMC an PMC+MEDLINE achieved the same average F1-score of 0.841 . This result also suggests that the full text of PMC is useful for BERTMeSH to achieve good performance for highly frequent MHs.

\subsubsection{Case Study}

Table 7 shows the results of a sample article with PMID $=31261512$ (PMCID=PMC6616313). The first five rows of Table 7 are PMID (PMCID), title, abstract, part of main text, and MHs. The last four rows are the predicted MHs by DeepMeSH, FullMesH, BERTMeSH (MEDLINE) and BERTMeSH (PMC+MEDLINE), where correctly predicted MHs are highlighted in bold. Among the four methods, BERTMeSH (PMC+MEDLINE) achieved the highest F-measure of 0.947, while Fmeasure of the other three methods were all around 0.75 . Specifically, three MHs, "Aged, 80 and over" , "ROC Curve" and "Young Adult" (all colore by red) were all correctly predicted by BERTMeSH (PMC+MEDLINE) but not by all other methods. We found that the descriptions most related with these three MHs cannot be seen in abstract, but appear in body text (in the Result section). For example, "The mean patient age was $55.4 \pm 14$. years with a range of 18 to 91 years;" in the Result section implies two MHs: "Aged, 80 and over" and "Young Adult". BERTMeSH could capture this information, while the other three methods could not use full text or could not capture the above semantics from full text efficiently. Overall this case study demonstrates the advantage of BERTMeSH over DeepMeSH and FullMeSH.

\subsection{Computation time}

In our experiments, we used a server with 2 Intel Xeon E5-2678 V3 $2.5 \mathrm{GHz}$ CPUs, 256G memory and 8 NVIDIA GTX 1080TI GPUs. Training BERTMeSH needed around 4 days, including pre-training, while FullMeSH needed around 7 days with a cluster server of six nodes, each being equipped with 128 GB RAM and two Intel XEON E5-4650 CPUs. Prediction by BERTMeSH needed around 5 minutes for 20,000 articles, while FullMeSH needed over 10 hours for 20,000 articles, mainly due to high computational cost of $k$-nearest neighbors.

\section{Discussion and Conclusion}

With the rapid growth of biomedical articles, automatic MeSH indexing with full text is becoming increasingly important. FullMeSH, only method of using full text for large-scale MeSH indexing had two serious problems: FullMeSH 1) uses LTR, which is laborious and time consuming, 2) can use only pre-determined sections in full text, limiting the advantage of using full text, and 3) ignores the whole MEDLINE database. To address these challenges, we have developed a computationally lighter model, BERTMeSH, which is flexible in section organization of full text, by using the state-of-the-art, deep contextual representation, BERT. Also BERTMeSH has pre-training, which allows BERTMeSH to use both MEDLINE (with only title and abstract) and PMC (with full text). Extensive experiments using 20K full text articles of PMC showed the efficiency, effectiveness and robustness of BERTMeSH over recent cutting-edge baselines. Interesting future work is to incorporate other recent techniques developed in the deep learning community to capture the interaction between different sections of the full text.

\section{References}

Aronson, A. et al. (2004). The NLM indexing initiative's Medical Text Indexer. Stud Health Technol Inform, 107(Pt 1), 268-272.

Burns, G., Li, X., and Peng, N. (2019). Building deep learning models for evidence classification from the open access biomedical literature. Database, 2019, baz034 Dai, S. et al. (2020). FullMeSH: improving large-scale MeSH indexing with full text. Bioinformatics, 36(5), 1533-1541.

Devlin, J. et al. (2019). BERT: Pre-training of deep bidirectional transformers for language understanding. In NAACL2019, pages 4171-4186.

$\mathrm{Gu}$, J. et al. (2013). Efficient semisupervised MEDLINE document clustering with MeSH-semantic and global-content constraints. IEEE Transactions on Cybernetics, $\mathbf{4 3}(4), 1265-1276$.

Huang, X. et al. (2011). Enhanced clustering of biomedical documents using ensemble non-negative matrix factorization. Information Sciences, 181(11), 2293-2302.

Jin, Q. et al. (2018). AttentionMesH: Simple, effective and interpretable automatic mesh indexer. In BioASQ2018, pages 47-56.

Kingma, D. P. and Ba, J. (2014). Adam: A method for stochastic optimization. $\operatorname{arXiv}$ preprint arXiv: 1412.6980

Lee, J. et al. (2020). BioBERT: pre-trained biomedical language representation mode for biomedical text mining. Bioinformatics, 36(4), 1234-1240.

Li, H. (2011). A short introduction to learning to rank. IEICE Transactions, 94-D(10), 1854-1862.

Liu, K. et al. (2015). MeSHLabeler: improving the accuracy of large-scale MeSH indexing by integrating diverse evidence. Bioinformatics, 31(12), i339-i347.

Lu, Z. et al. (2009). Evaluation of query expansion using MeSH in PubMed. Information retrieval, 12(1), 69-80.

Mao, Y. and Lu, Z. (2017). MeSH Now: automatic MeSH indexing at PubMed scale via learning to rank. Journal of biomedical semantics, $\mathbf{8}(1), 15$.

Mikolov, T. et al. (2013). Distributed representations of words and phrases and their compositionality. In NIPS2013, pages 3111-3119.

Mork, J. et al. (2017). 12 years on-is the NLM Medical Text Indexer still useful and relevant? Journal of biomedical semantics, $\mathbf{8}(1), 8$. 


\begin{tabular}{|c|c|}
\hline PMID (PMCID) & 31261512 (РMC6616313) \\
\hline Title & A cross-sectional study of the ambulatory central artery stiffness index in patients with hypertension. \\
\hline Abstract & $\begin{array}{l}\text { The present study aimed to investigate the characteristics of the ambulatory central artery stiffness index (AcASI) and its related } \\
\text { factors. The association between AcASI and the left ventricular mass index (LVMI), and other factors related to atherosclerosis were } \\
\text { explored.Patients with primary hypertension were enrolled into this study. Ambulatory central artery blood pressure (CABP) and } \\
\text { ambulatory brachial artery blood pressure (BABP) were assessed using a Mobil-O-Graph NG hemomanometer, whereas AcASI and } \\
\text { the ambulatory arterial stiffness index (AASI) were determined. LVMI was assessed by echocardiography.A total of } 136 \text { patients } \\
\text { with primary hypertension were enrolled from May } 2011 \text { to January } 2013 \text { in Beijing Hospital. AcASI was significantly associated } \\
\text { with AASI (r=0.879, P<.001). AcASI was significantly lower than AASI }(0.422 \pm 0.302 \text { vs } 0.482 \pm 0.270 ; \text { P }<.001) \text {. AcASI increased } \\
\text { with age, ambulatory brachial mean blood pressure (MBP), and fasting glucose. AcASI was significantly associated with office pulse } \\
\text { pressure (PP), ambulatory brachial PP, ambulatory central PP, and pulse wave velocity (PWV). AcASI, but not AASI, was significantly } \\
\text { associated with LVMI. Receiver operator characteristic analysis indicated that AcASI and AASI could may be a predictor of left } \\
\text { ventricular hypertrophy (LVH). Multiple regression analysis indicated that AcASI, chronic kidney disease, and hypertension course } \\
\text { were associated with LVMI, but AASI was not.AcASI, which is obtained from ambulatory CABP monitoring, could be a new marker } \\
\text { for the evaluation of atherosclerosis. AcASI may be stronger associated with LVH than AASI. }\end{array}$ \\
\hline Section (Results) & $\begin{array}{l}\text { After evaluating the data efficacy, } 136 \text { patients with primary hypertension were finally enrolled. The mean patient age was } 55.4 \pm 14.1 \\
\text { years with a range of } 18 \text { to } 91 \text { years; } 98 \text { participants were male }(72.1 \%) \text {. . . ROC analysis showed that AASI and AcASI were } \\
\text { associated with the LVH (Fig.2). Z test by using Delong method indicated that the discriminatory difference in the area under the } \\
\text { curve of AASI and AcASI was } 0.755 \text { and } 0.807 \text {, respectively ( } \mathrm{P}=.106) \text {. AcASI did not show superiority than AASI. ROC analysis of } \\
\text { AASI and AcASI in predicting LVH. ROC analysis evaluating the ability of AASI and AcASI index to predict the presence of LVH. } \\
\text { The discriminatory difference in area under the curve of AASI and AcASI was } 0.755 \text { and } 0.807 \text { respectively, which indicated those } 2 \\
\text { curves were not related. . . . . }\end{array}$ \\
\hline MHs & $\begin{array}{l}\text { Adolescent; Adult; Aged; Aged, } 80 \text { and over; Blood Pressure; Blood Pressure Monitoring, Ambulatory; Cross-Sectional Studies; } \\
\text { Echocardiography; Female; Humans; Hypertension; Hypertrophy, Left Ventricular; Male; Middle Aged; ROC Curve; Retrospective } \\
\text { Studies; Vascular Stiffness; Young Adult }\end{array}$ \\
\hline $\begin{array}{l}\text { DeepMeSH } \\
\text { F-score: } 0.750\end{array}$ & $\begin{array}{l}\text { Adult; Aged; Arteries; Blood Pressure; Cross-Sectional Studies; Female; Humans; Hypertension; Male; Middle Aged; } \\
\text { Hypertrophy, Left Ventricular; Blood Pressure Monitoring, Ambulatory; Vascular Stiffness; Pulse Wave Analysis }\end{array}$ \\
\hline $\begin{array}{l}\text { FullMesH } \\
\text { F-score: } 0.750\end{array}$ & $\begin{array}{l}\text { Adult; Aged; Blood Pressure; Brachial Artery; Cross-Sectional Studies; Female; Humans; Hypertension; Male; Middle Aged; } \\
\text { Hypertrophy, Left Ventricular; Blood Pressure Monitoring, Ambulatory; Vascular Stiffness; Pulse Wave Analysis }\end{array}$ \\
\hline $\begin{array}{l}\text { BERTMeSH } \\
\text { (MEDLINE) } \\
\text { F-score: } 0.765\end{array}$ & $\begin{array}{l}\text { Humans; Cross-Sectional Studies; Hypertension; Male; Female; Middle Aged; Vascular Stiffness; Aged; Hypertrophy, Left } \\
\text { Ventricular; Blood Pressure Monitoring, Ambulatory; Brachial Artery; Echocardiography; Blood Pressure; Pulse Wave Analysis; } \\
\text { Atherosclerosis; Adult }\end{array}$ \\
\hline $\begin{array}{l}\text { BERTMeSH } \\
\text { (PMC+MEDLINE } \\
\text { F-score: } 0.947\end{array}$ & $\begin{array}{l}\text { Humans; Hypertension; Middle Aged; Male; Female; Cross-Sectional Studies; Adult; Aged; Aged, } 80 \text { and over; Adolescent; } \\
\text { Blood Pressure Monitoring, Ambulatory; Hypertrophy, Left Ventricular; Vascular Stiffness; Retrospective Studies; Young } \\
\text { Adult; Blood Pressure; Brachial Artery; Echocardiography; ROC Curve; Pulse Wave Analysis }\end{array}$ \\
\hline
\end{tabular}

Mork, J. G., Jimeno-Yepes, A., and Aronson, A. R. (2013). The NLM Medical Text Indexer system for indexing biomedical literature. In BioASQ@ CLEF.

Peng, S. et al. (2016). DeepMeSH: deep semantic representation for improving large-scale MeSH indexing. Bioinformatics, 32(12), i70-i79.

Pennington, J., Socher, R., and Manning, C. (2014). GloVe: Global vectors for word representation. In EMNLP2014, pages 1532-1543.

Peters, M. et al. (2018). Deep contextualized word representations. In NAACL2018, pages 2227-2237.

Pillai, I., Fumera, G., and Roli, F. (2013). Threshold optimisation for multi-label classifiers. Pattern Recognition, 46(7), 2055-2065.

Sayers, E. W. et al. (2020). Database resources of the National Center for Biotechnology Information. Nucleic acids research, 48(D1), D9-D16.
Stokes, N. et al. (2009). Exploring criteria for successful query expansion in the genomic domain. Information retrieval, 12(1), 17-50.

Tsatsaronis, G. et al. (2015). An overview of the BIOASQ large-scale biomedica semantic indexing and question answering competition. BMC Bioinformatics, 16 , 138.

Tsoumakas, G. et al. (2013). Large-scale semantic indexing of biomedical publications at BioASQ. In BioASQ workshop.

Xun, G. et al. (2019). MeSHProbeNet: a self-attentive probe net for MeSH indexing. Bioinformatics, 35(19), 3794-3802.

Zhu, S. et al. (2009). Enhancing MEDLINE document clustering by incorporating MeSH semantic similarity. Bioinformatics, 25(15), 1944-1951. 